\title{
Preattentive detection of auditory memory representations in accumulative drinker
}

\author{
Phakkharawat Sittiprapaporn \\ Assistant Professor and Head, Brain Science and Engineering Innovation Research Group, School of Anti-Aging and \\ Regenerative Medicine, Mae Fah Luang University, Bangkok, Thailand, and Department of Anti-Aging Science, School \\ of Anti-Aging and Regenerative Medicine, Mae Fah Luang University, Bangkok, Thailand
}

Background: Chinese and English listeners did not show the same left-hemisphere (LH) lateralization as Thai listeners when making perceptual judgments of Thai tones. Although the left hemisphere is selectively employed for processing linguistic information irrespectively of acoustic cues or subtype of phonological unit, the right-hemisphere $(\mathrm{RH})$ is employed for prosody-specific cues. Aims and Objective: As both hemispheres are lateralized for speech and language, the objective of this study is to extend the investigation of how the preattentive processing of contour tone changes of Thai monosyllabic words in alcoholic drinkers. Materials and Methods: Forty healthy right-handed adults participated in this study. Results: This study found that both rising-to-falling and falling-to-rising tone changes perception elicited Mismatch Negativity (MMN) between 217-264 msec with reference to the standard-stimulus Event-Related Potentials (ERPs). The rising-to-falling and falling-to-rising tone changes elicited a strong MMN for both groups. Source localization was obtained in the Middle Temporal Gyrus (MTG) of the right hemisphere (RH) for both groups. Conclusion: Automatic detection of changes in contour tones is a useful index of language universal auditory memory traces.

\section{Access this article online}

Website:

http://nepjol.info/index.php/AJMS DOI: 10.3126/ajms.v11i1.226579 E-ISSN: 2091-0576 P-ISSN: $2467-9100$

Key words: Event-related potential; Sound; Tone; Contour tone; Mismatch negativity

\section{INTRODUCTION}

In earlier studies, ${ }^{1-3}$ Chinese and English listeners did not show the same left-hemisphere (LH) lateralization as Thai listeners when making perceptual judgments of Thai tones. In addition, Chinese and English listeners were asked to make perceptual judgments of Chinese tones, consonants, and vowels. Chinese listeners showed left-hemisphere (LH) lateralization for both suprasegmental and segmental phonological units. ${ }^{3}$ These earlier studies suggest that functional circuits engage in early, pre-attentive speech perception of either suprasegmental or segmental units in tone languages. While it seems indisputable that language is sub-served by left-hemisphere (LH) and right-hemisphere (RH) are lateralized for speech, language, or something else. Hypotheses proposed to account for functional hemispheric asymmetries can generally be classified as either cue dependent i.e., basic neural mechanism underlie processing of complex auditory stimuli regardless of linguistic relevance, ${ }^{4}$ or task dependent, i.e., specialized neural mechanisms exist that are activated only by speech. ${ }^{5}$

Previous Event-Related Potentials (ERPs) studies at a phonetic level demonstrated that the MMN was enhanced in Finnish subjects by their first-language (Finnish) phoneme prototype rather than a non-prototype (Estonian) ${ }^{6}$ and that the MMN for a vowel contrast in Finnish was not generated in native Hungarian speakers with no knowledge of Finnish, ${ }^{7}$ implying that the MMN reflects language-specific memory traces formed by early and extensive exposure to a first language. However, language-specific word-related MMN/MMF components at acoustic and phonetic levels remain to be investigated in future studies. The differences between these studies provide the impetus for future investigations of duration processing and temporal integration differences across language groups. Although the left hemisphere is selectively employed for processing linguistic information 
irrespectively of acoustic cues or subtype of phonological unit, the right hemisphere is employed for prosody-specific cues. ${ }^{5}$ The propose of the present study is, thus, to use both an auditory MMN component of event-related potential (ERP) recording and the standardized Low Resolution Electromagnetic Tomography (sLORETA) technique to measure the degree of cortical activation and to localize the brain area contributing to the scalp recorded auditory MMN component, respectively, during the passive oddball paradigm. Thus, the objective of this study is to investigate the pre-attentive processing of contour tone changes in Thai alcoholic drinkers compared to Thai non-alcoholic drinkers.

\section{MATERIALS AND METHODS}

\section{Participants}

Forty healthy right-handed adults with normal hearing and no known neurological disorders volunteered for participation: twenty non-alcoholic drinkers (Control group), aged 23-39 (mean 27.6 \pm 0.34 ) and twenty alcoholic drinkers (Target group), aged 23-29 (mean 31.18 \pm 1.07 ). The approval of the institutional committee on human research and written consent from each subject were obtained.

\section{Stimuli and procedure}

Stimuli consisted of two pairs of monosyllabic, Thai words. Speech stimuli were digitally generated and edited to have equal peak energy level in decibels SPL with the remaining data within each of the stimuli scaled accordingly using the Cool Edit Pro v. 2.0 (Syntrillium Software Corporation). The sound pressure levels of speech stimuli were then measured at the output of the earphones (E-A-RTONE 3A, 50 $\Omega$ ) in dBA using a Brüel and Kjaer 2230 sound-level meter. Pairs were designed to have similar long vowel duration. Two different stimuli were synthetically generated: Stimuli $1: / \mathrm{k}^{\mathrm{h}}$ aam/- falling tone; Stimuli 2: $/ \mathrm{k}^{\mathrm{h}}$ aam $/$ - rising. Five Thai native speakers listened to the synthesized words and evaluated them all as natural sounding.

The standard (S)/deviant (D) pairs for each trial, which was randomized across participants. The sounds were presented binaurally via headphones (Telephonic TDH-39-P) at $85 \mathrm{~dB}$. The inter-stimulus interval (ISI) was 1.25 second (offset-onset). Deviant stimuli appeared randomly among the standards at 10\% probability. Each experiment included 125 trials $(10 \% \mathrm{D})$. The stimuli were binaurally delivered using ePrime v. 3.0 software (Psychology Software Tools, Inc., Pittsburgh, PA, USA) via headphones (Telephonic TDH-39-P). EEG signal recording was time-locked to the onset of a word. Participants were instructed pay attention to the stimuli presented via headphones, and responded to the deviant stimuli by pressing the button.

\section{Electroencephalographic recording}

For EEG/ERP recording, the standard 20 locations of the 10-20 system, EEG, eego ${ }^{\text {TM }}$ (ANT Neuro, Hengelo, Natherlands), was recorded waveguard EEG caps (ANT Neuro, Hengelo, Natherlands) from 20 active electrodes (Fp1, Fp2, F7, F3, Fz, F4, T3, C3, Cz, C4, T4, T5, P3, Pz, $\mathrm{P} 4, \mathrm{~T} 6, \mathrm{O} 1, \mathrm{Oz}, \mathrm{O} 2)$ positioned according to the 10-20 International System of Electrode Placement, plus $\mathrm{Oz}$ and Ground were applied, pre-mounted in waveguard EEG caps. Reference electrodes were manually applied to left and right mastoids, where the Fp1 electrode were used for ocular artifact detection. Vertical eye movements were monitored at Fp1. EEG was amplified with a gain of 30,000 and filtered with a band pass of 0.1-30 Hz. EEGs were acquired as continuous signals and were subsequently segmented into epochs of $1 \mathrm{~s}$ (a $100 \mathrm{msec}$ pre-stimulus baseline and a $900 \mathrm{msec}$ post-stimulus epoch).

\section{EEG data processing}

The recordings were filtered and carefully inspected for eye movement and muscle artifacts. ERPs were obtained by averaging epoch, which started $100 \mathrm{~ms}$ before the stimulus onset and ended $900 \mathrm{~ms}$ thereafter; the -100-0 $\mathrm{ms}$ interval was used as a baseline. Epochs with voltage variation exceeding $\pm 100 \mu \mathrm{V}$ at any EEG channel were rejected from further analysis. The MMN was obtained by subtracting the response to the standard from that to the deviant stimulus. All responses were recalculated offline against average reference for further analysis.

\section{Spatial analysis}

The average MMN latency was defined as a moment of the global field power (GFP) with an epoch of 50-ms time window related stable scalp-potential topography. ${ }^{8}$ In the next step, standardized Low-resolution Electromagnetic Tomography (sLORETA) was applied to estimate the current source density distribution in the brain, which contributed to the electrical scalp field. ${ }^{8}$ Maps are computed with sLORETA. sLORETA computed the smoothest of all possible source configurations throughout the brain volume by minimizing the total squared Laplacian of source strengths. ${ }^{8}$

\section{Data analysis}

During the auditory stimulation, electric activity of the participants' brain was continuously recorded. The MMN was obtained by subtracting the response to the standard (rising-to-falling OR falling-to-rising) from that to the deviant (falling-to-rising OR rising-to-falling) stimulus. The statistical significance of MMN was tested with one sample $t$-test. 


\section{RESULTS}

The grand-averaged ERPs showed that both rising-tofalling and falling-to-rising tone changes perception elicited MMN between 217-264 ms with reference to the standardstimulus ERPs. The $t$-test comparing MMN amplitudes of the standard and deviant were statisticallysignificant (e.g., control group (-3.83 \pm 0.28$)$, target group $(-3.07 \pm 0.47)$; $\mathrm{t}(39)=1.73, p<0.05$ in rising-to-falling tone changes, and control group $(-2.99 \pm 0.75)$, target group $(-2.19 \pm 0.75)$; $\mathrm{t}(39)=1.63, p<0.05$ in falling-to-rising tone changes). The result showed that rising-to-falling and falling-to-rising tone changes elicited a strong MMN for both control and target groups (Table 1 and Figure 1).

Source localization analyses were performed using sLORETA $^{8}$. Table 2 demonstrates the xyz-values in Talairach space as calculated with sLORETA in the time window 217-264 msec. In rising-to-falling tone changes,

\begin{tabular}{|c|c|c|}
\hline \multirow[t]{2}{*}{ Tone changes } & \multicolumn{2}{|c|}{ Participants } \\
\hline & Control group & Target group \\
\hline Rising-to-Falling & $-3.83 \pm 0.28$ & $-3.07 \pm 0.47$ \\
\hline Falling-to-Rising & $-2.99 \pm 0.75$ & $-2.19 \pm 0.75$ \\
\hline
\end{tabular}

\begin{tabular}{|c|c|c|c|c|c|}
\hline \multirow[t]{2}{*}{ Tone changes } & \multicolumn{3}{|c|}{ Coordinates $(\mathrm{mm})$} & \multirow[t]{2}{*}{ BA } & \multirow[t]{2}{*}{$t$ values } \\
\hline & $x$ & $y$ & $z$ & & \\
\hline \multicolumn{6}{|l|}{ Rising-to-Falling } \\
\hline Control Group & 53 & -60 & 15 & 22 & 5.91 \\
\hline Target Group & 46 & -67 & 8 & 39 & 4.69 \\
\hline \multicolumn{6}{|l|}{ Falling-to-Rising } \\
\hline Control Group & 53 & -60 & 15 & 22 & 5.73 \\
\hline Target Group & 46 & -67 & 8 & 39 & 4.69 \\
\hline
\end{tabular}

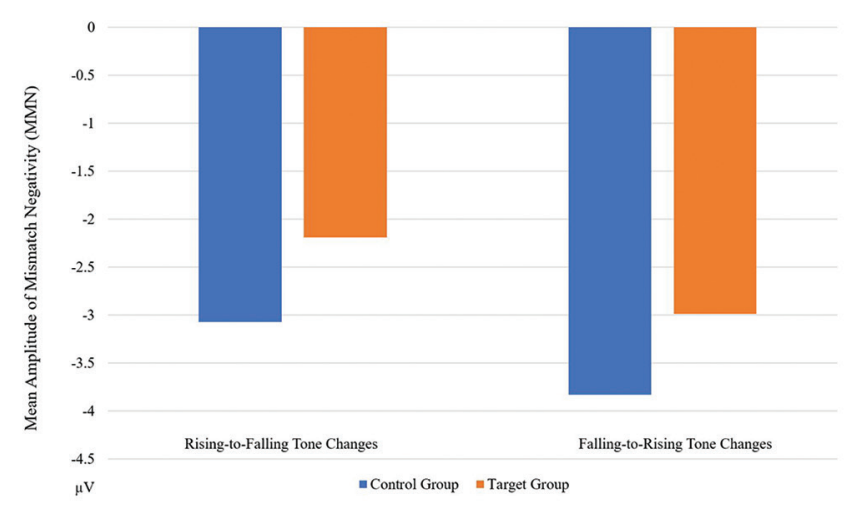

Figure 1: Mean amplitude $(\mu \mathrm{V}) \pm$ S.D. of MMN elicited by a contour tone change perception in control and target groups a single source was estimated to be located in the Middle Temporal Gyrus (MTG) of right hemisphere (RH) for both groups. In falling-to-rising tone changes, a single source was obtained in the Middle Temporal Gyrus (MTG) of right hemisphere $(\mathrm{RH})$ for both groups (Table 2, Figures 2, and Figure 3).

\section{DISCUSSION}

Both rising-to-falling and falling-to-rising tone changes perception elicited MMN between 217-264 ms with reference to the standard-stimulus ERPs. The risingto-falling and falling-to-rising tone changes elicited a strong MMN bilaterally for control and target groups. Source localization analyses performed using sLORETA demonstrates that sources were obtained in the Middle Temporal Gyrus (MTG) of the right hemisphere (RH) for both groups.

The same results were obtained in MEG studies using both tone and Japanese words., ${ }^{910}$ The present results parallel the finding in previous studies., ${ }^{910}$ The present study, the detection of tone changes was most likely acoustically driven rather than semantically driven, such that the stimuli were processed without any access to semantic information. The acoustic aspect in the absence of phonetic or higher-order properties may account for why target group had similar neuronal responses to control group. This suggests that at the point of stimulus disparity or thereafter, change detection of tone changes is somehow compromised. It is reasonable to speculate that the continued auditory processing required for the tone changes interferes with or masks the detection mechanism underlying the MMN. ${ }^{9}$ In the same way, the current findings showed similar results with previous tone studies that reported a clear MMN elicited by both duration increments and decrements ${ }^{11}$ and a larger MMN elicited by increments than decrements. ${ }^{12}$ The MMN component in the present study was also found to be more sensitive to control group compared to target group.

One might expect language-specific effects on the elicitation of the MMN in speech, since Thai is a tonal language and English is a stress-accent language. A tendency towards stronger MMN in target group is observed and support such an expectation in that statistically significant difference in MMN amplitudes was found between groups at an acoustic level. Previous ERP studies at a phonetic level demonstrated that the MMN was enhanced in Finnish subjects by their first-language (Finnish) phoneme prototype rather than a non-prototype (Estonian) ${ }^{1}$ and that the MMN for a vowel contrast in Finnish was not generated 

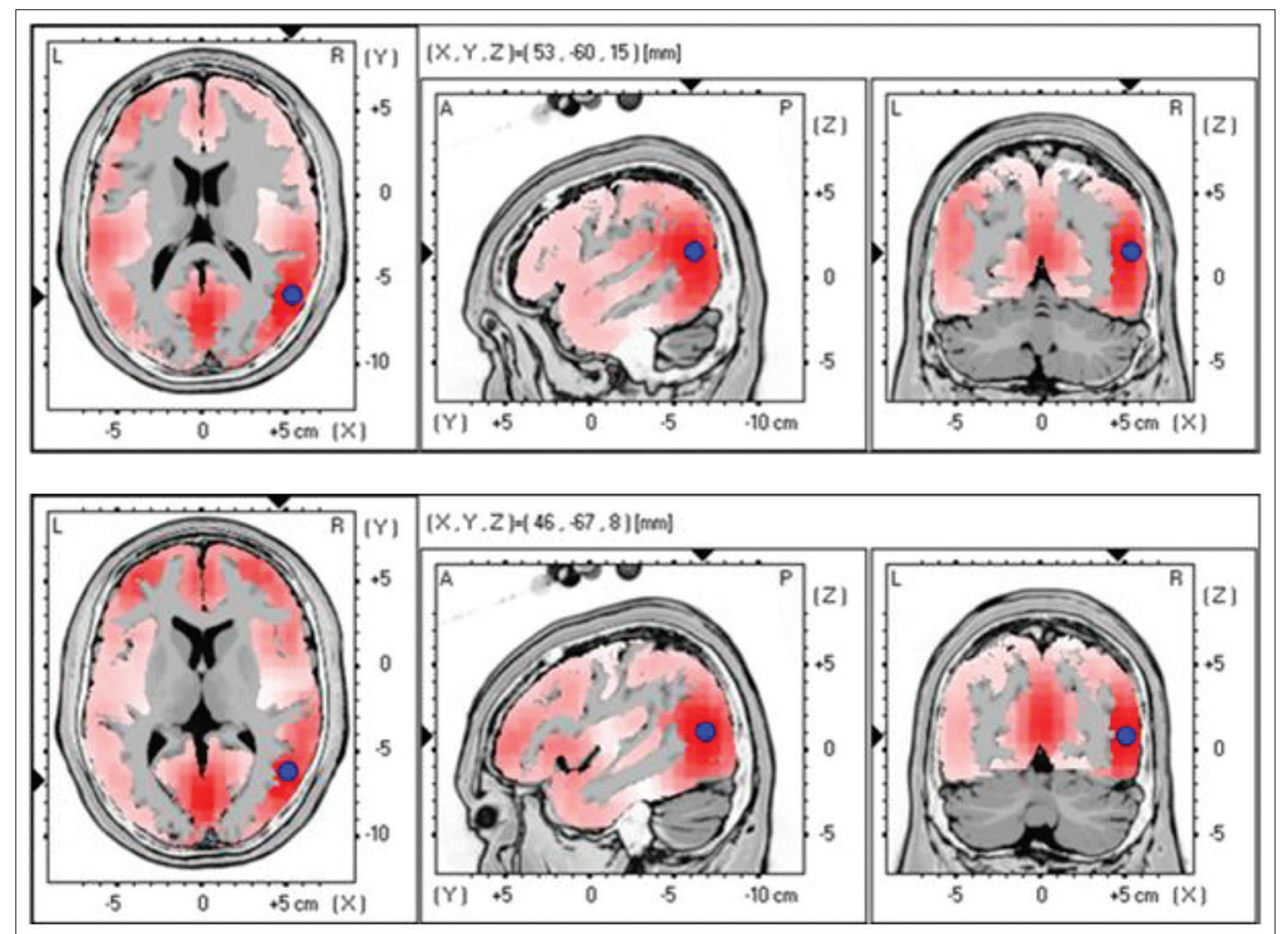

Figure 2: Graphical representation of the standardized Low-resolution Electromagnetic Tomography (sLORETA) $t$-statistic comparing the eventrelated potentials (ERPs) for mismatch negativity (MMN) responses at the time point of the individual peak over Fz for the rising-to-fallingtone changes of control group (top) and target group (bottom) activated in right hemisphere (RH). Red color indicates local maxima of increased electrical activity in an axial, a saggital and a coronal slice through the reference brain. Blue dots mark the center of significantly increased electric activity
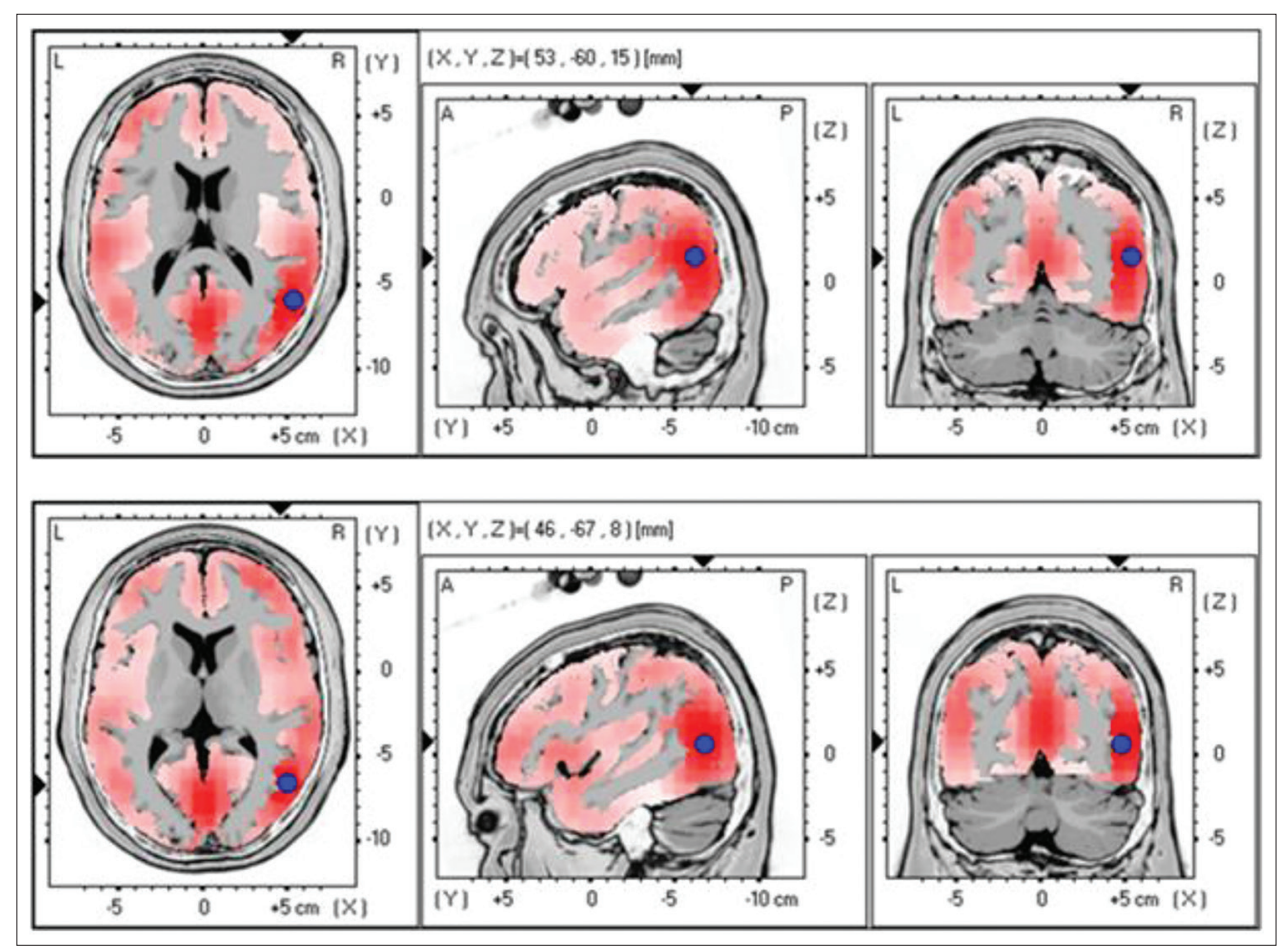

Figure 3: Graphical representation of the standardized Low-resolution Electromagnetic Tomography (sLORETA) $t$-statistic comparing the eventrelated potentials (ERPs) for mismatch negativity (MMN) responses at the time point of the individual peak over Fz for the falling-to-rising tone changes of control group (top) and target group (bottom) activated in right hemisphere (RH). Red color indicates local maxima of increased electrical activity in an axial, a saggital and a coronal slice through the reference brain. Blue dots mark the center of significantly increased electric activity

in native Hungarian speakers with no knowledge of Finnish, ${ }^{7}$ implying that the MMN reflects language-specific memory traces formed by early and extensive exposure to a first language. However, language-specific word-related 
MMN/MMF components at acoustic and phonetic levels in both groups remain to be investigated in future studies.

\section{CONCLUSION}

Both rising-to-falling and falling-to-rising tone changes perception elicited MMN between 217-264 ms with reference to the standard-stimulus ERPs. The rising-to-falling and falling-to-rising tone changes elicited a strong MMN bilaterally for control and target groups. Source localization analyses performed using sLORETA demonstrates that sources were obtained in the Middle Temporal Gyrus (MTG) of the right hemisphere (RH) for both groups. Automatic detection of changes in tone changes is a useful index of language universal auditory memory traces.

\section{ACKNOWLEDGEMENT}

This research was supported by research funding from Mae Fah Luang University grant (MFU-grant no. 61210320031), Mae Fah Luang University grant (Electroencephalogram Laboratory 2019), and Brain Science and Engineering Innovation Research Group, Mae Fah Luang University grant (2019) (MFU-grant no. 611U109005) and 2020, Thailand.

We thank all of the subjects involved in this research as main data provider of this studies.

\section{REFERENCES}

1. Gandour J. Aphasia in tone languages. In Coppens P, Basso A, Lebrun Y (Eds.), Aphasia in atypical populations (pp 117/141).
Hillsdale, NJ: Lawrence Erlbaum, 1998.

2. Gandour J, Wong D and Hsieh L. A Cross-linguistic PET Study of Tone Perception. Journal of Cognitive Neuroscience 2000; 12(1): 207-222.

3. Hsieh L, Gandour J, Wong D and Hutchins GD. Functional Heterogeneity of Inferior Gyrus is Shaped by Linguistic Experience. Brain and Language 2001; 10: 1-15.

4. Ivry $R$ and Roberson $L$. The two sides of perception. Cambridge, MA: MIT Press; 1998.

5. Imaizumi S Mori K, Kiritani S, Hosoi H and Tonoike M. Taskdependent laterality for cue decoding during spoken language processing. Neuro Report 1998; 9: 899-903.

6. Näätänen R. Attention and Brain Function. Lawrence Erlbaurn, Hillsdale, 1992.

7. Winkler I, Lehtokoski A, Alko P, Vainio M, Czigler I, Csepe V, et al. Pre-attentive detection of vowel contrasts utilizes both phonetic and auditory memory representations. Cognitive Brain Research 1999; 7: 357-369.

8. Pascual RD, Michel $\mathrm{CM}$ and Lehmann D. Low Resolution Electromagnetic Tomography: A New Method for Localizing Electrical Activity in the Brain. International Journal of Psychophysiolog 1994; 18: 49-65.

9. Inouchi M, Kubota M, Ferrari P and Roberts T. Neuromagnetic auditory cortex responses to duration and pitch changes in tones: cross-linguistic comparisons of human subjects in directions of acoustic changes. Neuroscience Letter 2002; 331: 138-142.

10. Inouchi M, Kubota M, Ferrari $P$ and Roberts T. Magnetic mismatch fields elicited by vowel duration and pitch changes in Japanese words in humans: comparison between native- and non-speakers of Japanese. Neuroscience Letter 2003; 353: 165-168.

11. Näätänen $R$, Paavilainen $P$ and Reinikainen $K$. Do event-related potentials to infrequent decrements in duration of auditory stimuli demonstrate a memory trace in man? Neuroscience Letter 1989; 107: 347-352.

12. Jaramillo $M, A l k u P$ and Paavilainen P. An event-related potential (ERP) study of duration changes in speech and non-speech sounds. Neuroreport 1990; 10: 3301-3305.

\footnotetext{
Authors Contribution:

PS- Concept and design of the study, statistically analyzed and interpreted, manuscript preparation, critical revision of the manuscript.

Work attributed to:

Brain Science and Engineering Innovation Research Group, School of Anti-Aging and Regenerative Medicine, Mae Fah Luang University, Thailand.

Orcid ID:

Dr. Phakkharawat Sittiprapaporn - (i) https://orcid.org/0000-0002-4103-9396

Source of Support: Mae Fah Luang University grant (MFU-grant no. 61210320031); Mae Fah Luang University grant (Electroencephalogram Laboratory 2019); Brain Science and Engineering Innovation Research Group, Mae Fah Luang University grant (2019) (MFU-grant no. 611U109005) and 2020, Thailand, Conflict of Interest: None.
} 